\title{
Real and complex symplectic structures. Application to concentration in degree for microfunctions at the boundary
}

\section{By Andrea D'Agnolo and Giuseppe ZAMPIERI}

(Received July 15, 1992)

\section{§1. Symplectic forms and Levi forms}

Let $X$ be a complex manifold of dimension $n, \pi: T^{*} X \rightarrow X$ the cotangent bundle, $\partial$ the holomorphic differential on $X$. Let $X^{\mathbf{R}}$ denote the real underlying manifold to $X$ and $d=\partial+\bar{\partial}$ its real differential. We shall always identify $T^{*}\left(X^{\mathbf{R}}\right) \widetilde{\rightarrow}\left(T^{*} X\right)^{\mathbf{R}}$. (If $\phi$ is a $C^{1}$ function on $X^{\mathbf{R}}$, the identification is given by $d \phi(x) \mapsto \partial \phi(x)$.) Let $\omega$ be the (complex) canonical 1-form on $T^{*} X, \sigma=\partial \omega$ the canonical 2-form, $H: T^{*} T^{*} X \rightarrow T T^{*} X$ the corresponding Hamiltonian isomorphism. We shall consider the induced one-forms $\omega^{\mathbf{R}}=2 \operatorname{Re} \omega, \omega^{\mathbf{I}}=2 \operatorname{Im} \omega$ and two-forms $\sigma^{\mathbf{R}}=2 \operatorname{Re} \sigma, \sigma^{I}=2 \operatorname{Im} \sigma$ on $T^{*} X^{\mathbf{R}}$.

Let $M$ be a real analytic hypersurface of $X$ defined by the equation $\phi(z)=0$ at $z_{0} \in M$. Let $p=\left(z_{0}, \partial \phi\left(z_{0}\right)\right)$, and set:

$$
\begin{aligned}
T_{z_{0}} M & =\left\{v \in T_{z_{0}} X ; \operatorname{Re}\langle v, \partial \phi\rangle=0\right\} \\
T_{z_{0}}^{\mathbf{C}} M & =T_{z_{0}} M \cap i T_{z_{0}} M=\left\{v \in T_{z_{0}} X ;\langle v, \partial \phi\rangle=0\right\} \\
\lambda_{M}(p) & =T_{p} T_{M}^{*} X \\
\lambda_{0}(p) & =T_{p} \pi^{-1} \pi(p) \\
v(p) & =\mathbf{C} H(\omega(p)) .
\end{aligned}
$$

The morphism

$$
\begin{aligned}
M & \rightarrow T_{M}^{*} X \\
z & \mapsto(z, \partial \phi(z)),
\end{aligned}
$$

induces the morphism

$$
\begin{aligned}
\psi: T_{z_{0}} M & \rightarrow \lambda_{M}(p) \\
v & \mapsto(v, \partial\langle\partial \phi, v\rangle+\partial\langle\bar{\partial} \phi, \bar{v}\rangle)
\end{aligned}
$$


by which $T_{z_{0}}^{\mathbf{C}} M$ is identified to $\{(v, \partial\langle\bar{\partial} \phi, \bar{v}\rangle) ;\langle\partial \phi, v\rangle=0\}$. Let $L_{M}$ be the Levi form of $M$ at $p$. Recall that, if $\left(z_{1}, \ldots, z_{n}\right)$ is a local system of coordinates at $z_{0}$, $L_{M}$ is the Hermitian form on $T_{z_{0}}^{\mathbf{C}} M$ represented by the matrix $\left(\partial_{i} \bar{\partial}_{j} \phi\left(z_{0}\right)\right)_{i, j}$. One immediately checks that:

$$
\psi^{*}\left(\left.\sigma\right|_{\psi\left(T_{z_{0}}^{\mathbf{C}} M\right)}\right)=L_{M}
$$

Let $S$ be a real analytic submanifold of $M$ with $\operatorname{codim}_{X} M=1, \operatorname{codim}_{M} S=r$. We shall set:

$$
\gamma(S, p)=\operatorname{dim}_{\mathbf{C}}\left(\lambda_{S}(p) \cap i \lambda_{S}(p) \cap \lambda_{0}(p)\right) .
$$

One says that $S \times{ }_{M} T_{M}^{*} X$ is regular when $\left.\omega\right|_{S \times{ }_{M} T_{M}^{*} X}\left(=\left.\frac{i}{2} \omega^{\mathbf{I}}\right|_{S \times_{M} T_{M}^{*} X}\right) \neq 0$.

Proposition 1.1. The following assertions are equivalent:

$$
\begin{aligned}
& S \times{ }_{M} T_{M}^{*} X \quad \text { is regular at } p ; \\
& \operatorname{dim}^{\mathbf{R}}\left(\lambda_{S}(p) \cap \nu(p)\right)=1 .
\end{aligned}
$$

Proof. Let $S$ be locally defined by the equations $\phi_{i}=0(i=1, \ldots, r+1)$, where $\phi_{1} \cong \phi$ is a local equation for $M$, and recall that $p=\left(z_{0}, \partial \phi\left(z_{0}\right)\right)$. We have:

$$
\psi^{*}\left(\omega^{\mathbf{I}}\right)=-\operatorname{Re}(i \partial \phi)
$$

Thus:

$$
\begin{aligned}
S \times{ }_{M} T_{M}^{*} X \quad \text { is regular } & \Leftrightarrow i \frac{\partial \phi}{\partial z}\left(z_{0}\right) \notin\left(T_{S}^{*} X\right)_{z_{0}} \\
& \Leftrightarrow \operatorname{dim}_{\mathbf{R}}\left(\lambda_{S}(p) \cap \nu(p)\right)=1 .
\end{aligned}
$$

REMARK 1.2. Let $\operatorname{codim}_{X} S=2, \operatorname{codim}_{X} M=1$. Then $S \times{ }_{M} T_{M}^{*} X$ is regular iff $\gamma(S, p)=0$ (i.e. $S$ is "generic").

We shall assume from now on that

$$
T_{M}^{*} X \quad \text { is I-symplectic }
$$

i.e. that $\left.\sigma^{\mathbf{I}}\right|_{\lambda_{M}}$ is non-degenerate. This is equivalent to $\lambda_{M}(p) \cap i \lambda_{M}(p)=\{0\}$ or else to

$$
L_{M} \text { is non-degenerate, }
$$

(cf [S1]). In fact via $\psi$ one may easily identify $\lambda_{M}(p) \cap i \lambda_{M}(p)$ with the null-space of $L_{M}\left(\mathrm{cf}\left[\mathrm{D}^{\prime} \mathrm{A}-\mathrm{Z} 1\right]\right)$. 
Corresponding to the non-degenerate forms $\sigma^{\mathbf{I}}$ and $L_{M}$ we may thus consider two Hamiltonian isomorphisms $H^{\mathbf{I}}$ and $\widetilde{H}^{\mathbf{I}}$ :

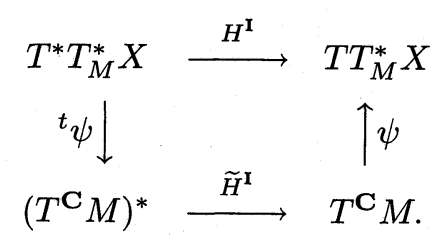

Consider the morphism $\pi_{M}^{*}: T_{z_{0}}^{*} M \rightarrow T_{p}^{*} T_{M}^{*} X$ induced by the projection $\pi_{M}$ : $T_{M}^{*} X \rightarrow M$.

Proposition 1.3. For $\theta \in T_{z_{0}}^{*} M$, we have

$$
\left.H^{\mathbf{I}}\left(\pi_{M}^{*}(\theta)\right)\right|_{\psi\left(T^{\mathbf{C}} M\right)}=\psi(v)
$$

(in the identification $\lambda_{M}(p) \widetilde{\rightarrow} \lambda_{M}^{*}(p)$ given by $w \mapsto \sigma(w, \cdot)$ ), where $v \in T_{z_{0}}^{\mathbf{C}} M$ is the unique solution of

$$
\partial\langle\bar{\partial} \phi, \bar{v}\rangle=\left.\frac{i}{2} \theta\right|_{T^{\mathbf{C}} M}
$$

(in the identification $T^{\mathbf{C}} M \widetilde{\rightarrow} T^{\mathbf{C}} M^{*}$ given by $v \mapsto\langle v, \cdot\rangle$ ).

Proof. The vectors $v$ which satisfy (1.4) must verify for every $u \in T_{z_{0}}^{\mathrm{C}} M$ :

$$
\begin{aligned}
\frac{1}{2}[\langle\theta, u\rangle+\langle\bar{\theta}, \bar{u}\rangle] & =\sigma(\psi(v), \psi(u)) \\
& =-[i\langle\partial\langle\bar{\partial} \phi, \bar{v}\rangle, u\rangle-i\langle\partial\langle\bar{\partial} \phi, \bar{u}\rangle, v\rangle] \\
& =-[i\langle\partial\langle\bar{\partial} \phi, \bar{v},\rangle, u\rangle+\overline{i\langle\partial\langle\bar{\partial} \phi, \bar{v}\rangle, u\rangle}]
\end{aligned}
$$

hence:

$$
\left.\operatorname{Re}\left\langle\frac{i}{2} \theta, \cdot\right\rangle\right|_{T^{\mathbf{C}} M}=\left.\operatorname{Re}\langle\partial\langle\bar{\partial} \phi, \bar{v}\rangle, \cdot\rangle\right|_{T^{\mathbf{C}} M}
$$

Reasoning in the same way for $i u$ we get the conclusion.

Let us remark now that (when (1.3) holds):

$$
\operatorname{dim}_{\mathbf{R}}\left(\lambda_{S}(p) \cap \nu(p)\right)=1 \quad \Leftrightarrow \quad i H^{\mathbf{I}}\left(\pi^{*}(p)\right) \notin \lambda_{S}(p),
$$

where $\pi^{*}: T_{z_{0}}^{*} X \rightarrow T_{p}^{*} T^{*} X$ denotes the map associated to $\pi: T^{*} X \rightarrow X$. We may then correspondingly rephrase the equivalent conditions of Proposition 1.1. We also have 
Proposition 1.4. Let $S \subset M \subset X$ with $\operatorname{codim}_{X} M=1$ and with $L_{M}$ being positive or negative definite at $p$. Then

$$
S \times{ }_{M} T_{M}^{*} X \quad \text { is regular involutive } \Rightarrow \gamma(S, p)=0 .
$$

Proof. Let $S$ be locally defined by the equations $\phi_{i}=0(i=1, \ldots, r+1)$, where $\phi_{1} \cong \phi$ is a local equation for $M$. We have already seen that:

$$
S \times{ }_{M} T_{M}^{*} X \quad \text { is regular } \Leftrightarrow i \partial \phi \notin \mathbf{R} \partial \phi_{1}+\cdots+\mathbf{R} \partial \phi_{r+1}
$$

$$
\left.\Leftrightarrow \partial \phi_{2}\right|_{T^{\mathbf{C}} M}, \ldots,\left.\partial \phi_{r+1}\right|_{T^{\mathbf{C}} M} \text { are } \mathbf{R} \text {-independent. }
$$

Let $v_{i}$ solve (1.4) for $\theta_{i}=\partial \phi_{i}$. Let $\chi$ and $\psi$ be functions on $M$ which vanish on $S$ and let $u, v \in \bigoplus_{i} \mathbf{R} v_{i}$ be such that: $\psi(u)=H^{\mathbf{I}}\left(\pi_{M}^{*}(d \chi)\right), \psi(v)=H^{\mathbf{I}}\left(\pi_{M}^{*}(d \psi)\right)$. Owing to Proposition 1.3, we have:

$$
\{\chi, \psi\}_{\mathbf{I}}=-i L_{M}(\bar{u} \wedge v)
$$

$\left(\{\cdot, \cdot\}_{\mathbf{I}}\right.$ denoting the Poisson bracket). Hence:

$$
\{\chi, \psi\}_{\mathbf{I}}=0 \forall \chi, \psi \text { (i.e. } S \times{ }_{M} T_{M}^{*} X \text { is involutive) } \Rightarrow u \neq i v \forall u, v \text {. }
$$

(In fact if $v=i u$, then $\sigma(H u, H v)=i \sigma^{\mathbf{I}}(H u, i H u)=-2 i L_{M}(\bar{u} \wedge u) \neq 0$ since $L_{M}$ is definite $(>0$ or $<0)$.) We also remark that

$$
v \mapsto\left\langle\partial\langle\bar{\partial} \phi, \bar{v}\rangle,\left.\cdot\right|_{T^{\mathbf{C}} M}\right. \text { is injective, }
$$

due to (1.4). By (1.7), (1.8), this implies that $\left.\partial \phi_{i}\right|_{T^{\mathrm{C}}}, i \geq 2$ are C-independent. This is in turn equivalent to the fact that $\partial \phi_{i}, i \geq 1$ are $\mathbf{C}$-independent.

\section{§2. Degenerate R-Lagrangian submanifolds}

Let $X$ be a complex manifold of dimension $n$, and let $\bar{X}$ be the complex conjugate manifold to $X$. We shall identify $X^{\mathbf{R}}$ to the diagonal $X \times{ }_{X} \bar{X}$. The manifold $X \times \bar{X}$ is then a natural complexification of $X^{\mathbf{R}}$.

Let $M$ be a $C^{\omega}$-hypersurface of $X^{\mathbf{R}}$ defined at $z_{0} \in M$ by the equation $\phi(z)=$ 0 , and set $p=\left(z_{0}, \partial \phi\left(z_{0}\right)\right)$. We shall identify $\mathbf{C} \bigotimes_{\mathbf{R}} T_{z_{0}} M$ with $\left\{(u, \bar{v}) \in T_{z_{0}} X \times\right.$ $\left.T_{z_{0}} \bar{X} ;\langle\partial \phi, u\rangle+\langle\bar{\partial} \phi, \bar{v}\rangle=0\right\}$ and consider:

$$
\begin{aligned}
\psi^{\mathbf{C}}: \mathbf{C} \otimes_{\mathbf{R}} T_{z_{0}} M & \rightarrow \lambda_{M}(p)+i \lambda_{M}(p) \\
(u, \bar{v}) & \mapsto(u ; \partial\langle\partial \phi, u\rangle+\partial\langle\bar{\partial} \phi, \bar{v}\rangle)
\end{aligned}
$$


Let $S \subset M$ be a submanifold with $\operatorname{codim}_{M} S=r$. Consider the null space

$$
N S\left(\left.L_{M}\right|_{T_{z_{0}}} ^{\mathbf{C} S}\right)=\left\{v \in T_{z_{0}}^{\mathbf{C}} S ; \partial\langle\bar{\partial} \phi, \bar{v}\rangle \in \pi^{*}\left(\left(T_{S}^{*} X\right)_{z_{0}}\right)\right\}
$$

Using $T X \hookrightarrow T X \times{ }_{T X} T \bar{X}$ and $\psi$, we get

Proposition 2.1. We have an identification

$$
\lambda_{S}(p) \cap i \lambda_{S}(p) \cong N S\left(\left.L_{M}\right|_{T_{z_{0}}^{\mathbf{C}} S}\right) \bigoplus\left(\lambda_{S}(p) \cap i \lambda_{S}(p) \cap \lambda_{0}(p)\right)
$$

Proof. We shall often omit the indices $z_{0}$ and $p$ in the following. The projection $\mathcal{R}: H \pi^{*}\left(T_{S}^{*} X+i T_{S}^{*} X\right) \longrightarrow H \pi^{*}\left(T_{S}^{*} X\right)$ is well defined modulo $\lambda_{S} \cap i \lambda_{S} \cap \lambda_{0}$. It is easy to check that (cf also [D'A-Z1]):

$$
\begin{aligned}
& \frac{\lambda_{S}}{\lambda_{S}} \cap i \lambda_{S} \cap \lambda_{S} \cap \lambda_{0} \\
& \quad=\left\{\psi^{\mathbf{C}}(v)-2 \mathcal{R}(\partial\langle\bar{\partial} \phi, \bar{v}\rangle) ; v \in T^{\mathbf{C}} S, \partial\langle\bar{\partial} \phi, \bar{v}\rangle \in H \pi^{*}\left(T_{S}^{*} X+i T_{S}^{*} X\right)\right\} .
\end{aligned}
$$

Then (2.2) follows at once.

We suppose all through this section that $T_{M}^{*} X$ is I-symplectic and that $S \times{ }_{M}$ $T_{M}^{*} X$ is a regular involutive submanifold of $T_{M}^{*} X$. We also set $\Sigma=S \times{ }_{M} T_{M}^{*} X$ and define $\widetilde{\Sigma}$ to be the union of the complexifications of the bicharacteristic leaves of $\Sigma$. This is a germ of R-Lagrangian submanifold of $T^{*} X^{\mathbf{R}}$.

Proposition 2.2. We have an identification

$$
T_{p} \widetilde{\Sigma} \cap i T_{p} \widetilde{\Sigma} \cong \psi\left(\left\{v \in T_{z_{0}} S+i T_{z_{0}} S ; \partial\langle\bar{\partial} \phi, \bar{v}\rangle \in H \pi^{*}\left(T_{S}^{*} X_{z_{0}}+i T_{S}^{*} X_{z_{0}}\right)\right\}\right)
$$

Proof. We have by definition

$$
T \widetilde{\Sigma}=T \Sigma+i T \Sigma^{\perp}
$$

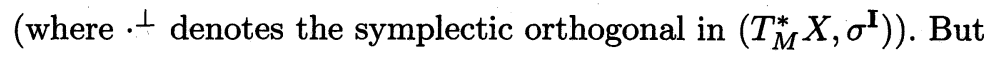

$$
T \Sigma^{\perp}=\psi\left(\left\{u \in T^{\mathbf{C}} M \cap T S ;\left.\left.\partial\langle\bar{\partial} \phi, \bar{u}\rangle\right|_{T^{\mathbf{C}} M} \in i H \pi^{*}\left(T_{S}^{*} X\right)\right|_{T^{\mathbf{C}} M}\right\}\right) .
$$

Then (2.3) follows immediately.

Proposition 2.3. Under the above assumptions, we have

$$
\operatorname{dim}_{\mathbf{C}}\left(T_{p} \tilde{\Sigma} \cap i T_{p} \widetilde{\Sigma}\right)=r
$$


Proof. We have $T \Sigma \cap i T \Sigma=0$ (due to the fact that $T_{M}^{*} X$ is I-symplectic). It follows:

$$
\begin{aligned}
T \widetilde{\Sigma} \cap i T \widetilde{\Sigma} & =\left(T \Sigma+i T \Sigma^{\perp}\right) \cap\left(T \Sigma^{\perp}+i T \Sigma\right) \\
& =T \Sigma^{\perp}+i T \Sigma^{\perp}=\mathbf{C} \bigotimes_{\mathbf{R}} T \Sigma^{\perp}
\end{aligned}
$$

For $v \in T \widetilde{\Sigma}+i T \widetilde{\Sigma}$ (resp. $v \in T \Sigma+i T \Sigma$, resp. $v \in \lambda_{S}+i \lambda_{S}$ ) let us denote by $v^{c_{T \widetilde{\Sigma}}}$ (resp. $v^{c_{T \Sigma}}$, resp. $v^{c_{\lambda_{S}}}$ ) the "conjugate" with respect to $T \widetilde{\Sigma}$ (resp. $T \Sigma$, resp. $\left.\lambda_{S}\right)$. This is well defined modulo $T \widetilde{\Sigma} \cap i T \widetilde{\Sigma}=(T \widetilde{\Sigma}+i T \widetilde{\Sigma})^{\perp}(\operatorname{resp} . T \Sigma \cap i T \Sigma=\{0\}$, resp. $\left.\lambda_{S} \cap i \lambda_{S}=\left(\lambda_{S}+i \lambda_{S}\right)^{\perp}\right)$. We remark now that $T \widetilde{\Sigma}+i T \widetilde{\Sigma}=T \Sigma+i T \Sigma$ and thus

$$
\left.\left.\sigma\left(v, w^{c_{T} \widetilde{\Sigma}}\right)\right|_{v, w \in(T \widetilde{\Sigma}+i T \widetilde{\Sigma}) \cap \lambda_{0}} \sim \sigma\left(v, w^{c_{T \Sigma}}\right)\right|_{v, w \in(T \Sigma+i T \Sigma) \cap \lambda_{0}}
$$

(where " $"$ means equivalence in signature and rank). On the other hand:

$$
\left\{\begin{array}{l}
\psi^{\mathbf{C}}\left(\overline{T^{\mathbf{C}} S}\right)+\left(\lambda_{S} \cap \lambda_{0}\right)^{\mathbf{C}}=\left(\lambda_{S}+i \lambda_{S}\right) \cap \lambda_{0} \\
\psi^{\mathbf{C}}\left(\overline{T^{\mathbf{C}} S}\right) \cap\left(\lambda_{S} \cap \lambda_{0}\right)^{\mathbf{C}}=\lambda_{S} \cap i \lambda_{S} \cap \lambda_{0}
\end{array}\right.
$$

and

$$
(T \Sigma+i T \Sigma) \cap \lambda_{0}=\psi^{\mathbf{C}}\left(\overline{T^{\mathbf{C}} S}\right) \bigoplus\left(\lambda_{S} \cap \lambda_{0}\right)^{\mathbf{C}}
$$

(where ${ }^{\mathbf{C}}=\cdot+i$. and where we make the identification $\overline{T^{\mathbf{C}} S} \hookrightarrow\{0\} \times T \bar{X}$ ). It follows:

$$
\left.\left.\sigma\left(v, w^{c_{T \Sigma}}\right)\right|_{v, w \in(T \Sigma+i T \Sigma) \cap \lambda_{0}} \sim \sigma\left(v, w^{c_{\lambda_{S}}}\right)\right|_{v, w \in\left(\lambda_{S}+i \lambda_{S}\right) \cap \lambda_{0}} .
$$

Let $\tau$ denote the inertia index for a triple of Lagrangian planes in the sense of [K-S1] and [K-S2]. We recall that $\frac{1}{2} \tau\left(T \widetilde{\Sigma}, i T \widetilde{\Sigma}, \lambda_{0}\right)$ and $n-\operatorname{dim}_{\mathbf{R}}\left(T \widetilde{\Sigma} \cap \lambda_{0}\right)+$ $2 \operatorname{dim}_{\mathbf{C}}\left(T \widetilde{\Sigma} \cap i T \widetilde{\Sigma} \cap \lambda_{0}\right)-\operatorname{dim}_{\mathbf{C}}(T \widetilde{\Sigma} \cap i T \widetilde{\Sigma})$ are respectively the signature and the $\operatorname{rank}$ of $\left.\sigma\left(v, w^{c_{T} \widetilde{\Sigma}}\right)\right|_{v, w \in(T \widetilde{\Sigma}+i T \widetilde{\Sigma}) \cap \lambda_{0}}$. In the same way $\frac{1}{2} \tau\left(\lambda_{S}, i \lambda_{S}, \lambda_{0}\right)$ and $n-1-$ $r+2 \gamma(S)-\operatorname{dim}_{\mathbf{C}}\left(\lambda_{S} \cap i \lambda_{S}\right)$ are signature and rank of $\left.\sigma\left(v, w^{c_{\lambda_{S}}}\right)\right|_{v, w \in \lambda_{S} \cap i \lambda_{S} \cap \lambda_{0}}$ due to [D'A-Z1]. By (2.7), (2.8) the above signatures and ranks have to coincide. In particular

$$
\begin{aligned}
& \operatorname{dim}_{\mathbf{R}}\left(T \widetilde{\Sigma} \cap \lambda_{0}\right)+\operatorname{dim}_{\mathbf{C}}(T \widetilde{\Sigma} \cap i T \widetilde{\Sigma})-2 \operatorname{dim}_{\mathbf{C}}\left(T \widetilde{\Sigma} \cap i T \widetilde{\Sigma} \cap \lambda_{0}\right) \\
& =1+r+\operatorname{dim}_{\mathbf{C}}\left(\lambda_{S} \cap i \lambda_{S}\right)-2 \gamma(S)
\end{aligned}
$$

We also notice that

$$
\begin{aligned}
T \widetilde{\Sigma} \cap i T \widetilde{\Sigma} \cap \lambda_{0} & =\left.H \pi^{*}\left(T_{S}^{*} X \cap i T_{S}^{*} X\right)\right|_{T^{\mathbf{c}} M} \\
& \cong H \pi^{*}\left(T_{S}^{*} X \cap i T_{S}^{*} X\right) \\
& =\lambda_{S} \cap i \lambda_{S} \cap \lambda_{0}
\end{aligned}
$$


Thus by using (2.10) and (2.6) in (2.9) we get at once the following statement.

TheOREM 2.4. We have

$$
\operatorname{dim}_{\mathbf{R}}\left(T_{p} \widetilde{\Sigma} \cap \lambda_{0}\right)=1+\operatorname{dim}_{\mathbf{C}}\left(\lambda_{S}(p) \cap i \lambda_{S}(p)\right) .
$$

\section{§3. A vanishing theorem for generalized microfunctions at the boundary}

Let $X$ be an open subset of $\mathbf{C}^{n}$, and let $M$ be a $C^{\omega}$-hypersurface of $X$. We denote by $D^{b}(X)$ the derived category of the category of complexes of sheaves with bounded cohomology. We denote by $\mathcal{O}_{X}$ the sheaf of holomorphic functions on $X$ and we will consider its microlocalization along $M, \mu_{M}\left(\mathcal{O}_{X}\right)\left(\mu_{M}\right.$ being the Sato microlocalization functor). For any $C^{\omega}$-submanifold $W$ of $X^{\mathbf{R}}$, we shall similarly define $\mu_{W}\left(\mathcal{O}_{X}\right)$.

Let $M$ be defined in local coordinates by $\phi(z)=0$ and let $p=\left(z_{0}, \partial \phi\left(z_{0}\right)\right)$ with $z_{0} \in M$. Let $s^{ \pm}(M, p)$ denote the numbers of eigenvalues $\gtrless 0$ for the Levi form $L_{M}$. Let $S$ be a $C^{\omega}$-submanifold of $M$ with $\operatorname{codim}_{M} S=r$. We shall denote by $s^{ \pm}(S, p)$ the corresponding numbers of eigenvalues for $\left.L_{M}\right|_{T^{\mathrm{C}} S}$ (cf [D'A-Z1]). Set now $\Lambda=T_{M}^{*} X, \Sigma=S \times{ }_{M} T_{M}^{*} X$ and assume that $\Lambda$ is I-symplectic and $\Sigma$ is regular involutive in $\Lambda$. Let us recall that the latter hypothesis implies that $\operatorname{dim}_{\mathbf{R}}\left(\lambda_{S}(p) \cap \nu(p)\right)=1$ (recall that $\nu$ denotes the Euler vector field), and moreover, if $M$ is the boundary of a strictly pseudoconvex or pseudoconcave domain, it implies that $\gamma(S, p):=\operatorname{dim}_{\mathbf{C}}\left(\lambda_{S}(p) \cap i \lambda_{S}(p) \cap \lambda_{0}(p)\right)=0$.

Define $\tilde{\Sigma}$ to be the union of the complexifications of the bicharacteristic leaves of $\Sigma$. Let us assume

$$
\delta(S, p):=\operatorname{dim}_{\mathbf{C}}\left(\lambda_{S}(p) \cap i \lambda_{S}(p)\right) \text { is constant with respect to } p .
$$

Let us remark that if (3.1) holds, then by (2.11) there exists $W$ with $S \subset W \subset X$ and with $\operatorname{codim}_{X} W=1+\delta(S, p)$ such that $\widetilde{\Sigma}=T_{W}^{*} X$.

We also know from [K-S1, Proposition 11.3.5] that

$$
\mu_{M}\left(\mathcal{O}_{X}\right) \text { is concentrated in degree } 1+s^{-}(M, p) \text {. }
$$

(In fact the assumptions of that Proposition are fulfilled since, $T_{M}^{*} X$ being Isymplectic, $s^{-}(M, p)$ is constant.) Let $S \subset M \subset X$, $\operatorname{codim}_{X} M=1$, $\operatorname{codim}_{M} S=r$. Our result goes as follows.

Theorem 3.1. Let $T_{M}^{*} X$ be $\mathbf{I}$-symplectic, let $S \times{ }_{M} T_{M}^{*} X$ be regular involutive in $T_{M}^{*} X$, and assume (3.1) to hold. Then

$$
H^{j} \mu_{W}\left(\mathcal{O}_{X}\right)=0 \text { for } j \neq 1+\delta(S, p)+s^{-}(S, p)-\gamma(S, p)
$$


Proof. (We shall often omit the indices $p$ or $z_{0}=\pi(p)$ in the following.) We perform a contact transformation $\chi$ in $T^{*} X$ such that

$$
\begin{cases}T_{M}^{*} X \rightarrow T_{M^{\prime}}^{*} X & \operatorname{codim}_{X} M^{\prime}=1, s^{-}\left(M^{\prime}\right)=0 \\ \Sigma \rightarrow S^{\prime} \times{ }_{M^{\prime}} T_{M^{\prime}}^{*} X & \operatorname{codim}_{M^{\prime}} S^{\prime}=r, \gamma\left(S^{\prime}\right)=0 \\ T_{W}^{*} X(=\tilde{\Sigma}) \rightarrow T_{W^{\prime}}^{*} X & \operatorname{codim}_{X} W^{\prime}=1 .\end{cases}
$$

(The fact that $\gamma\left(S^{\prime}\right)=0$ in the second line follows from Proposition 1.4. This, together with $\left.N S\left(L_{M^{\prime}}\right)\right|_{T^{\mathrm{c}} S_{S^{\prime}}}=\{0\}$. (cf. §2), gives $\delta\left(S^{\prime}\right)=0$ due to Proposition 2.1. This implies codim $W^{\prime}=1$ in the third line of (3.4), due to Theorem 2.4.) Let $s^{0}(S):=\delta(S)-\gamma(S)$; thus $s^{+}(S)+s^{-}(S)+s^{0}(S)=n-1-r-\gamma(S)=$ $\operatorname{codim}_{T^{\mathrm{C}}}^{\mathrm{C}} T^{\mathrm{C}} S$. We have

$$
s^{+}\left(W^{\prime}\right) \equiv n-1-r, s^{0}\left(W^{\prime}\right) \equiv r
$$

(since $\left.\delta\left(W^{\prime}\right)=\operatorname{dim}_{\mathbf{C}}(\widetilde{\Sigma} \cap i \widetilde{\Sigma})=r\right)$. In particular $s^{-}\left(W^{\prime}\right)=0$ and hence $T_{W^{\prime}}^{*} X$ is the (exterior) conormal to the boundary of a (weakly) pseudoconvex domain and therefore by the results of [H1]:

$$
\mu_{W^{\prime}}\left(\mathcal{O}_{X}\right) \text { is concentrated in degree } 1 .
$$

By (3.5) we have $\frac{1}{2} \tau\left(\lambda_{W^{\prime}}, i \lambda_{W^{\prime}}, \lambda_{0}\right)=n-1-r$. We recall from $\S 2$ :

$$
\begin{aligned}
\left.\sigma\left(v, w^{c_{\lambda_{W}}}\right)\right|_{v, w \in\left(\lambda_{W}+i \lambda_{W}\right) \cap \lambda_{0}} & \left.\sim \sigma\left(v, w^{c_{T \Sigma}}\right)\right|_{(T \Sigma+i T \Sigma) \cap \lambda_{0}} \\
& \left.\sim \sigma\left(v, w^{c_{\lambda_{S}}}\right)\right|_{\left(\lambda_{S}+i \lambda_{S}\right) \cap \lambda_{0}}
\end{aligned}
$$

In particular $s^{ \pm}(W)=s^{ \pm}(S)$. It follows

$$
\begin{aligned}
\frac{1}{2} \tau\left(\lambda_{W}, i \lambda_{W}, \lambda_{0}\right) & =s^{+}(W)+s^{-}(W)-2 s^{-}(W) \\
& =n-1-\delta(S)-r+2 \gamma(S)-2 s^{-}(S) .
\end{aligned}
$$

Let

$$
\begin{aligned}
d_{W, W^{\prime}}=\frac{1}{2}\left[\operatorname{codim} W^{\prime}-\operatorname{codim} W\right. & -\frac{1}{2} \tau\left(\lambda_{W^{\prime}}, i \lambda_{W^{\prime}}, \lambda_{0}\right) \\
+ & \left.\frac{1}{2} \tau\left(\lambda_{W}, i \lambda_{W}, \lambda_{0}\right)\right]
\end{aligned}
$$


We know from [K-S1, Proposition 11.2.8] that the contact transformation $\chi$ can be quantized to an isomorphism

$$
\chi_{*}\left(\mu_{W}\left(\mathcal{O}_{X}\right)\right) \cong \mu_{W^{\prime}}\left(\mathcal{O}_{X}\right)\left[d_{W, W^{\prime}}\right]
$$

But

$$
\begin{aligned}
d_{W, W^{\prime}} & =\frac{1}{2}\left[1-(1+\delta(S))-(n-1-r)+\left(n-1-\delta(S)+2 \gamma(S)-2 s^{-}(W)\right]\right. \\
& =-\delta(S)+\gamma(S)-s^{-}(S) .
\end{aligned}
$$

Lemma 3.2. The following are equivalent

$$
\begin{aligned}
& s^{-}(M, p)-s^{-}(S, p)=\delta(S)-\gamma(S, p) \\
& s^{+}(M, p)-s^{+}(S, p)=r-\gamma(S, p)
\end{aligned}
$$

Proof. We have $\left(s^{+}(M)+s^{-}(M)\right)-\left(s^{+}(S)+s^{-}(S)\right)=r+\delta(S)-2 \gamma(S)$.

Let $S \subset M \subset X, \operatorname{codim}_{X} M=1, \operatorname{codim}_{M} S=r$.

Proposition 3.3. Let $T_{M}^{*} X$ be I-symplectic, let $S \times{ }_{M} T_{M}^{*} X$ be regular involutive in $T_{M}^{*} X$, let (3.1) hold, and let

$$
s^{-}(M, p)-s^{-}(S, p)=\delta(S, p)-\gamma(S, p) .
$$

We may then define a natural morphism

$$
\mu_{W}\left(\mathcal{O}_{X}\right) \rightarrow \mu_{M}\left(\mathcal{O}_{X}\right)
$$

Proof. We may find a contact transformation on $T^{*} X$

$$
\left\{\begin{array}{l}
T_{M}^{*} X \rightarrow T_{\mathbf{R}^{n}}^{*} \mathbf{C}^{n}, \\
\Sigma \rightarrow W^{\prime} \times \mathbf{R}^{n} T_{\mathbf{R}^{n}}^{*} \mathbf{C}^{n}, \quad W^{\prime} \subset \mathbf{R}^{n},
\end{array}\right.
$$

and hence $T_{W}^{*} X \rightarrow T_{W^{\prime}}^{*} \mathbf{C}^{n}$. Thus the natural morphism

$$
\mu_{W^{\prime}}\left(\mathcal{O}_{X}\right) \rightarrow \mu_{\mathbf{R}^{n}}\left(\mathcal{O}_{X}\right)
$$

induces (3.16) (via quantization of $\chi$ as in (3.11), (3.12)) if and only if $d_{W^{\prime}, W}=$ $d_{\mathbf{R}^{n}, M}$ i.e.

$$
1+\delta(S)-(n+r)-\frac{1}{2} \tau\left(\lambda_{W}, i \lambda_{W}, \lambda_{0}\right)=1-n-\frac{1}{2} \tau\left(\lambda_{M}, i \lambda_{M}, \lambda_{0}\right)
$$


i.e.

$$
\delta(S)-r-(n-1-\delta(S)-r+2 \gamma(S))+2 s^{-}(S)=-(n-1)+2 s^{-}(M) .
$$

The latter is in turn equivalent to (3.15). triangle:

We define now $\mu_{\Lambda \backslash \widetilde{\Sigma}}\left(\mathcal{O}_{X}\right)$ in $D^{b}(X)$ to be the third term of a distinguished

$$
\mu_{W}\left(\mathcal{O}_{X}\right) \rightarrow \mu_{M}\left(\mathcal{O}_{X}\right) \rightarrow \mu_{\Lambda \backslash \widetilde{\Sigma}}\left(\mathcal{O}_{X}\right) \stackrel{+1}{\rightarrow}
$$

Let $S \subset M \subset X, \operatorname{codim}_{X} M=1, \operatorname{codim}_{M} S=r$.

TheOREM 3.4. Let $T_{M}^{*} X$ be I-symplectic, let $S \times{ }_{M} T_{M}^{*} X$ be regular involutive, let (3.1) hold, and assume:

$$
s^{-}(M, p)-s^{-}(S, p)=\delta(S, p)-\gamma(S, p)
$$

Then

$$
H^{j} \mu_{\Lambda \backslash \widetilde{\Sigma}}\left(\mathcal{O}_{X}\right)=0 \text { for } j \neq 1+s^{-}(M, p)
$$

Proof. We have $1+\delta(S)-\gamma(S)+s^{-}(S)=1+s^{-}(M)$. Hence by Proposition $3.1, \mu_{W}\left(\mathcal{O}_{X}\right)$ and $\mu_{M}\left(\mathcal{O}_{X}\right)$ are both concentrated in degree $1+s^{-}(M)$. Moreover owing to Proposition 3.3 we have a morphism

$$
H^{1+s^{-}(M)} \mu_{W}\left(\mathcal{O}_{X}\right) \rightarrow H^{1+s^{-}(M)} \mu_{W}\left(\mathcal{O}_{X}\right),
$$

which is injective due to [S2].

REMARK 3.5. We will give in [D'A-M-Z] the following extension of the previous results. Let $\mu(p):=\lambda_{M}(p) \cap i \lambda_{M}(p)$ and $\delta(M, p):=\operatorname{dim}_{\mathbf{C}}(\mu(p))$. The preceding results can be extended to the following situation:

$$
\left\{\begin{array}{l}
M \text { is generic } \\
\delta(M, p) \text { is constant for } p \in T_{M}^{*} X \\
T_{p} \Sigma \supset \mu(p) \\
T_{p} \Sigma^{\mu(p)} \text { is regular involutive in } \lambda_{M}^{\mu(p)}(p) .
\end{array}\right.
$$

In this frame, the results on involuti vity and "genericity" of $\S 1$ still hold, and we also have

$$
\begin{aligned}
\operatorname{dim}_{\mathbf{C}}(T \widetilde{\Sigma} \cap i T \widetilde{\Sigma}) & =r+\delta(M), \\
\operatorname{dim}_{\mathbf{C}}\left(T \widetilde{\Sigma} \cap i T \widetilde{\Sigma} \cap \lambda_{0}\right) & =\operatorname{dim}_{\mathbf{C}}\left(\lambda_{S} \cap i \lambda_{S} \cap \lambda_{0}\right), \\
\operatorname{dim}_{\mathbf{R}}\left(T \widetilde{\Sigma} \cap \lambda_{0}\right) & =1+\delta(S)-\delta(M) .
\end{aligned}
$$


In particular if $\delta(S)$ is constant in $p$, then there exists $W \subset X$ with $\widetilde{\Sigma}=T_{W}^{*} X$. We still have

$$
\begin{aligned}
s^{ \pm}(W) & =s^{ \pm}(S) \\
s^{0}(W) & =r+\delta(M)-\gamma(S) \\
& =s^{0}(M)+\operatorname{codim}_{W} S .
\end{aligned}
$$

Thus repeating step by step the proof of Theorem 3.1 we get the following statement:

Assume that (3.20) is fulfilled and that $\delta(S)$ is constant in $p$ (and hence $\widetilde{\Sigma}=$ $\left.T_{W}^{*} X\right)$. Then

(i) $\quad H^{j} \mu_{W}\left(\mathcal{O}_{X}\right)=0$ for $j \neq 1+s^{-}(S)+\delta(S)-\delta(M)-\gamma(S)$

(ii) If moreover $s^{-}(M)-s^{-}(S)=\delta(S)-\delta(M)-\gamma(S)$ then there is a natural morphism

$$
\mu_{W}\left(\mathcal{O}_{X}\right) \rightarrow \mu_{M}\left(\mathcal{O}_{X}\right)
$$

and if one defines $\mu_{\Lambda \backslash \widetilde{\Sigma}}\left(\mathcal{O}_{X}\right)$ as in (3.17), one has

$$
H^{j} \mu_{\Lambda \backslash \widetilde{\Sigma}}\left(\mathcal{O}_{X}\right)=0 \quad \text { for } j \neq 1+s^{-}(M)
$$

\section{References}

[ A-H ] A. Andreotti and C.D. Hill, E.E. Levi convexity and the Hans Lewy problem. Part II: Vanishing theorems, Ann.Scuola Norm. Sup. Pisa, 26 (1972), 747-806.

[D'A-Z1] A. D'Agnolo and G. Zampieri, Levi's forms of higher codimensional submanifolds, Rend. Mat. Acc. Lincei, s. 92 (1991), 29-33.

[D'A-Z2] A. D'Agnolo and G. Zampieri, Generalized Levi forms for microdifferential systems, "D-modules and microlocal geometry", M. Kashiwara, T. Monterio Fernandes, P. Schapira eds., Walter de Gruyter Publ., 1992.

[ H1 ] L. Hörmander, An introduction to complex analysis in several complex variables, Van Nostrand, Princeton N.J., 1966.

[ H2 ] L. Hörmander, The analysis of linear partial differential operators, Springer Grundlehren der Math., 1974.

[ K-S1 ] M. Kashiwara and P. Schapira, Microlocal study of sheaves, Astérisque, 128 (1985).

[ K-S2 ] M. Kashiwara and P. Schapira, Sheaves on manifolds, Springer Grundlehren der Math., 292 (1990).

[ S1 ] P. Schapira, Condition de positivité dans une variété symplectique complexe. Applications à l'étude des microfunctions, Ann. Sci. École Norm. Sup., 14 (1981), 121-139.

[ S2 ] P. Schapira, Front d'onde analytique au bord II, Sem. E.D.P. Ecole Polyt. Exp. XIII (1986).

[S-K-K] M. Sato, M. Kashiwara and T. Kawai, Hyperfunctions and pseudodifferential equations, Springer Lecture Notes in Math., 287 (1973), 265-529.

[ $\mathrm{T}$ ] J.M. Trépreau, Systèmes différentiels à caractéristiques simples et structures réellescomplexes (d'après Baouendi-Trèves et Sato-Kashiwara-Kawaï), Sém. Bourbaki, $595(1981-82)$. 
[ $\mathrm{Sj}$ ] J. Sjostrand, Singularités analytiques microlocales, Astérique, 95 (1982).

[ K-K ] M. Kashiwara and T. Kawai, On the boundary value problem for elliptic systems of linear differential equations I, Proc. Japan Acad., 48 (1972), 712-715; Ibid. II, 49 (1973), 164-168.

\author{
ANDREA D'AgNOLO \\ DÉP. DE MATHÉMATIQUES \\ UNIVERSITÉ PARIS-NORD \\ 93430 Villetaneuse, France \\ GIUSEPPE ZAMPIERI \\ DIP. DI MATEMATICA \\ UNIVERSITÀ DI PADOVA \\ VIA Belzoni 7, 35131 Padova, Italy
}

